\title{
The Lymphatic System: A New Focus on its Role in Metabolism and Metabolic Disorders
}

\author{
Junji Yokozawa ${ }^{1,2}$, Ko Nagino ${ }^{1}$, Yu Sasaki ${ }^{1}$, Akiko Matsuda ${ }^{1}$, Yoshiyuki Ueno ${ }^{1}$ and Sumio Kawata ${ }^{1,3^{*}}$ \\ ${ }^{1}$ Department of Gastroenterology, Faculty of Medicine, Yamagata University, Yamagata 990-9585, Japan \\ ${ }^{2}$ Department of Integrative Genomics, Tohoku Medical Megabank Organization, Tohoku University, Sendai, Japan \\ ${ }^{3}$ Hyogo Prefectural Nishinomiya Hospital, Nishinomiya 662-0918, Japan
}

"Corresponding author: Sumio Kawata' Department of Gastroenterology, Faculty of Medicine, Yamagata University, Hyogo Prefectural Nishinomiya Hospital, Nishinomiya, Japan, Tel: +81 23-628-4006; E-mail: sumio_kawata@pref.hyogo.lg.jp

Rec date: June 30, 2014, Acc date: 30 August, 2014, Pub date: 01 September, 2014

Copyright: ( ) Yokozawa J, et al. This is an open-access article distributed under the terms of the Creative Commons Attribution License, which permits unrestricted use, distribution, and reproduction in any medium, provided the original author and source are credited.

Keywords: Glucose-dependent insulinotropic polypeptide; Glucagon-like peptide-1; Lymphatic system; Metabolism; Metabolic disorders

\section{Commentry}

Lymphatic vessels not only play a physiological role in maintenance of homeostasis and the immune response together with blood vessels, but also participate in the development of pathological states such as inflammation and cancer metastasis [1]. In addition, mesenteric lymphatic vessels have a specific function, including absorption of long-chain fatty acids from the small intestine. Several recent studies have shown that the lymph circulation plays an important role in not only lipid metabolism [2] but also carbohydrate metabolism [3], and that morphological and functional impairment of the lymphatic system may be related to the pathophysiology of metabolic syndrome [4] and type 2 diabetes mellitus [5].

Prox1 is a master gene essential for lymphatic vascular development. Recently, Harvey et al. [6] reported that impairment of lymph angiogenesis promoted by Prox 1 haploinsufficiency led to adult-onset obesity with an increased insulin level and mildly impaired glucose tolerance, although food intake and exercise were decreased. The lymphatic vessels of Prox $1^{+/-}$mice, in which Prox 1 had been inactivated by in-frame insertion of a $\beta$-galactosidase-neomycin resistance cassette in the $\mathrm{N}$-terminal part of the gene, were dilated, and lymphatic vascular mispatterning was most marked in the intestine and mesentery. To confirm that the lymphatic dysfunction observed in Prox $1^{+/-}$mice was the primary cause of the obesity phenotype, the authors generated a conditional knock-out mouse strain, Prox $1^{\Delta /+}$ Conditional Prox $1^{\Delta /+}$ mice with excision of the region spanning exons 2 and 3 of Prox 1 and showing partial lymphatic-specific removal of Prox 1 activity were significantly heavier than their wild-type counterparts at 12 weeks of age, and gained further weight to become obese. The levels of insulin and leptin were increased in these mice, although not to a statistically significant degree. Together with evident chyle-promoted adipocyte differentiation in vitro, the authors concluded that the lymphatic vascular defects in this murine model acted as the initial trigger of obesity.

Incretins, glucose-dependent insulinotropic polypeptide (GIP) and glucagon-like peptide-1 (GLP-1), are produced in enteroendocrine K cells and L cells, respectively, in the intestine in response to ingestion of carbohydrate and fat. Both GIP and GLP-1 stimulate insulin secretion from pancreatic $\beta$-cells, accounting for $\sim 50-70 \%$ of postprandial insulin secretion from the pancreas. Recently, it has been shown that intestinal lymph is rich in GIP [7] and GLP-1 [8], and studies using the lymph fistula rat model have demonstrated that the carbohydrate and lipid contents of food affect the secretion of GIP and GLP-1 into intestinal lymph vessels [2,3]. The incretin concentrations in lymph are significantly higher than in portal or systemic blood $[7,8]$. These findings suggest that intestinal lymph flow plays an important role in insulin secretion and glucose metabolism after meals.

Recently, Nagino et al. [9] examined changes in blood glucose level and insulin secretion resulting from blockage of mesenteric lymph flow by ligation of the thoracic duct in rats. One group of male Sprague-Dawley rats underwent ligation of the mesenteric lymph duct above the cistern (ligation group), and the other underwent a sham operation (sham group). At 2 weeks after the operation, the ligation group showed a significant decrease in the plasma glucose concentration at $120 \mathrm{~min}$ and a significant increase in the plasma insulin concentration by more than 2 -fold at 15 min during an oral glucose tolerance test (OGTT). The active form of GLP-1 showed a significantly higher level at $90 \mathrm{~min}(1.7$-fold $)$ and $120 \mathrm{~min}$ (2.5-fold) during the OGTT. The ratios of $\beta$-cell area/acinar cell area and $\beta$-cell area/islet area, and also $\beta$-cell proliferation assessed by Ki-67 immunostaining, were significantly higher in the ligation group than in the sham group. The insulin content per unit wet weight of pancreas was also significantly increased in the ligation group. The authors concluded that mesenteric lymph duct flow has a role in glucose metabolism.

It is interesting that proliferation of $\beta$-cells in islets was observed in the rats with mesenteric lymph duct ligation, possibly due to retention of mesenteric lymphatic fluid. Incretins are reported to stimulate proliferation of $\beta$-cells by induction of cyclin D1 via activation of phosphatidylinositol 3-kinase and protein kinase A. Incretins are secreted into the intestinal lymphatics and subsequently flow into the blood stream, while these hormones are known to regulate insulin activity via degradation with dipeptidyl peptidase-IV (DPP-IV). Recently, it has been reported that DPP-IV activity is much lower in lymph than in blood $[7,8]$. It still remains unexplained why rats subjected to lymph duct ligation should show an increase of GLP-1. It is possible that secretion of incretins is influenced by intestinal lymphatic flow, for example through changes in their production by intestinal $\mathrm{K}$ and $\mathrm{L}$ cells.

The relationship between obesity and lymphatic function is poorly understood. Data for humans are controversial: one study found impairment of lymphatic function in patients with morbid obesity [10], whereas another found that lymph flow in normal-weight and obese individuals were similar [11]. Blum et al. [12] investigated whether diet-induced obesity affected the morphology and function of the lymphatic system. In vivo analysis using high-resolution near 
infrared (NIR) imaging showed that the collecting lymphatic vessels of mice fed a high-fat diet were enlarged. Moreover, the function of collecting lymphatic vessels was negatively correlated with body weight. The contraction frequencies of collecting lymphatic vessels and their response to mechano stimulation were significantly reduced in three different mouse strains fed a high-fat diet (HFD). In K14VEGF-C mice, the HFD resulted in reduced spread of the tracer within dermal lymphatic vessels. These observations imply that adipose tissue expansion can lead to lymphatic dysfunction.

Impaired glucose metabolism seems to contribute to functional and morphological abnormality of the lymph circulation. Dermal lymphatic vessels play important roles in tissue fluid homeostasis, lipid absorption, and immune surveillance. Type 2 diabetes is well known to be associated with micro vascular injury through impairment of wound healing, subsequently resulting in infections and chronic ulcers in the skin. However, it has not been investigated whether the lymphatic vasculature is involved in such manifestations of dermal injury. Recently, Haemmerle et al. [5] demonstrated that lymphatic vessel density in the dermis was enhanced in patients with diabetes. The authors carried out transcriptional profiling of ex-vivo isolated lymphatic endothelial cells (LECs) and then identified 160 genes whose expression differed between type 2 diabetic and non-diabetic LECs. The deregulated genes, including those encoding TNF-aand chemokine CXCL10, were functionally related to inflammation, lymphatic vessel remodeling, lymphangiogenesis, and transport of lipid and small molecules. In addition, the authors demonstrated that paracrine cross-talk enhancing the recruitment of macrophages to LECs was one of the pathophysiological processes involved. In type 2 diabetes, this type of abnormality in lymphatic vessels could lead to a decrease of tissue fluid and reduced immune drainage, finally resulting in chronic skin inflammation.

Metabolic syndrome is well-known to lead not only ischemic cardiovascular diseases, but also diabetes and stroke. It has been suggested that the pathophysiology of metabolic syndrome widely overlaps the lymphatic function of adipose tissues [13]. Also, secretion of adipokines, such as TNF- $\alpha$, MCP-1 and leptin, secreted by human adipose tissues in vivo is partitioned between capillary and lymphatic transport [14]. Recently, Zawieja et al. [4] observed altered contractility of mesenteric lymphatics, reduced lymphatic muscle function, and vessel remodeling in an animal model of metabolic syndrome. These findings suggested that proper lymphatic flow was impaired in metabolic syndrome. Further studies are needed to clarify whether metabolic syndrome affects the lymphatic system, or vice versa.

Many metabolic disorders are associated with dysfunction and pathology of blood vessels, and therefore similar defects of the closely related lymphatic vessels would also be expected to occur in humans. Recent studies of lymphatic system dysfunction and pathology seem to have provided a new insight into the pathophysiology of metabolic syndrome and type 2 diabetes mellitus.

\section{References}

1. Alitalo K, Tammela T, Petrova TV (2005) Lymphangiogenesis in development and human disease. Nature 438: 946-953.

2. Yoder SM, Yang Q, Kindel TL (2009) Stimulation of incretin secretion by dietary lipid: is it dose dependent? Am J Physiol Gastrointest Liver Physiol 297: G299-G305.

3. Yoder SM, Yang Q, Kindel TL (2010) Differential responses of the incretin hormones GIP and GLP-1 to increasing doses of dietary carbohydrate but not dietary protein in lean rats. Am J Physiol Gastrointest Liver Physiol 299: G476-485.

4. Zawieja SD, Wang $\mathrm{W}, \mathrm{Wu} \mathrm{X}$ (2012) Impairment in the intrinsic contractility of mesenteric collecting lymphatics in a rat model of metabolic syndrome. Am J Physiol Heart Circ Physiol 302: H643-653.

5. Haemmerle M, Keller T, Egger G (2013) Enhanced lymph vessel density, remodeling, and inflammation are reflected by gene expression signatures in dermal lymphatic endothelial cells in type 2 diabetes. Diabetes 62: 2509-2529.

6. Harvey NL, Srinivasan RS, Dillard ME (2005) Lymphatic vascular defects promoted by Prox1 haploinsufficiency cause adult-onset obesity. Nat Genet 37: 1072-1081.

7. Lu WJ, Yang Q, Sun W (2008) Using the lymph fistula rat model to study the potentiation of GIP secretion by the ingestion of fat and glucose. Am J Physiol Gastrointest Liver Physiol 294: G1130-1138.

8. D'Alessio D, Lu W, Sun W (2007) Fasting and postprandial concentrations of GLP-1 in intestinal lymph and portal plasma: evidence for selective release of GLP-1 in the lymph system. Am J Physiol Regul Integr Comp Physiol 293: R2163-R2169.

9. Nagino K, Yokozawa J, Sasaki Y (2012) Increased secretion of insulin and proliferation of islet $\beta$-cells in rats with mesenteric lymph duct ligation. Biochem Biophys Res Commun 425: 266-272.

10. Greene AK, Grant FD, Slavin SA (2012) Lower-extremity lymphedema and elevated body-mass index. N Engl J Med 366: 2136-2137.

11. Vasileiou AM, Bull R, Kitou D (2011) Oedema in obesity; role of structural lymphatic abnormalities. Int J Obes (Lond) 35: 1247-1250.

12. Blum SB, Karaman S, Proulx ST (2014) Chronic high-fat diet impairs collecting lymphatic vessel function in mice. PLoS One 9: e94713.

13. Harvey NL (2008) The link between lymphatic function and adipose biology. Ann NY Acad Sci 1131: 82-88.

14. Miller NE, Michel CC, Nanjee N (2011) Secretion of adipokines by human adipose tissue in vivo: partitioning between capillary and lymphatic transport. Am J Physiol Endocrinol Metab 301: E659-E667. 